\title{
101 In circumscribed labyrinthitis
}

A Symptoms may be precipitated by auricular movement.

B Autoinflation is the most effective method of eliciting the fistula sign.

C In a weakly positive fistula sign nystagmus without vertigo is elicited.

D If caused by atticoantral CSOM is an indication for conservative management.

E The hearing deficit will not recover.

\section{In syphilitic labyrinthitis}

A The highest incidence occurs in the late stage of neurosyphilis.

B The fluorescent treponemal antibody absorbed test (FTA-ABS) is the least reliable serological test.

C Microscopic endolymphatic hydrops is a common feature.

D Vestibular reactions are completely lost.

E Fibrous bands may connect the medial surface of the stapes footplate to the membranous labyrinth.

\section{Pathophysiology of Menière's Disease}

A Sympathetic overactivity may play a role.

B There is gross dilatation of the scala vestibuli.

C Fibrous bands may connect the footplate to the distended saccule.

D Ruptures of the membranous labyrinth have been postulated as the cause of acute attacks.

E Sodium and potassium levels in the endolymph are grossly abnormal. 